\title{
Generalized operator for Alexander integral operator
}

\section{H. Özlem Güney}

Dicle University, Faculty of Science,

Department of Mathematics, 21280 Diyarbakır-Turkey

email: ozlemg@dicle.edu.tr
Shigeyoshi Owa

Honorary Professor

"1 Decembrie 1918" University

Alba Iulia, Romania

email: shige21@ican.zaq.ne.jp

Abstract. Let $T_{n}$ be the class of functions $f$ which are defined by a power series

$$
f(z)=z+a_{n+1} z^{n+1}+a_{n+2} z^{n+2}+\ldots
$$

for every $z$ in the closed unit disc $\overline{\mathbb{U}}$. With $m$ different boundary points $z_{s},(s=1,2, \ldots, m)$, we consider $\alpha_{m} \in e^{i \beta} A_{-j-\lambda} f(\mathbb{U})$, here $A_{-j-\lambda}$ is the generalized Alexander integral operator and $\mathbb{U}$ is the open unit disc. Applying $A_{-j-\lambda}$, a subclass $B_{n}\left(\alpha_{m}, \beta, \rho ; j, \lambda\right)$ of $T_{n}$ is defined with fractional integral for functions $f$. The object of present paper is to consider some interesting properties of $f$ to be in $B_{n}\left(\alpha_{m}, \beta, \rho ; j, \lambda\right)$.

\section{Introduction}

Let $T_{n}$ be the class of functions

$$
f(z)=z+\sum_{k=n+1}^{\infty} a_{k} z^{k}, \quad n \in \mathbb{N}=\{1,2,3, \ldots\}
$$

2010 Mathematics Subject Classification: 30C45, 30C50

Key words and phrases: analytic function, Alexander integral operator, fractional integral, gamma function, Miller and Mocanu lemma 
that are analytic in the closed unit disc $\overline{\mathbb{U}}=\{z \in \mathbb{C}:|z| \leq 1\}$. For $f \in T_{n}$, J.W.Alexander [2] had defined the following the Alexander integral operator $A_{-1} f(z)$ given by

$$
A_{-1} f(z)=\int_{0}^{z} \frac{f(t)}{t} d t=z+\sum_{k=n+1}^{\infty} \frac{a_{k}}{k} z^{k} .
$$

The above the Alexander integral operator was applied for some subclasses of analytic functions in the open unit disc $\mathbb{U}=\{z \in \mathbb{C}:|z|<1\}$ by M.Acu [1] and by K. Kugita et al. [4].

For the above the Alexander integral operator $A_{-1} f(z)$, we consider

$$
A_{-j} f(z)=A_{-j+1}\left(A_{-1} f(z)\right)=z+\sum_{k=n+1}^{\infty} \frac{a_{k}}{k^{j}} z^{k}, \quad j \in \mathbb{N}
$$

where $A_{0} f(z)=f(z)$.

From the various definitions of fractional calculus of $f \in T_{n}$ (that is, fractional integrals and fractional derivatives) given in the literature, we would like to recall here the following definitions for fractional calculus which were used by Owa [7] and Owa and Srivastava [8].

Definition 1 The fractional integral of order $\lambda$ for $\mathrm{f} \in \mathrm{T}_{\mathrm{n}}$ is defined by

$$
D_{z}^{-\lambda} f(z)=\frac{1}{\Gamma(\lambda)} \int_{0}^{z} \frac{f(t)}{(z-t)^{1-\lambda}} d t, \quad(\lambda>0)
$$

where $f$ is an analytic function in a simply-connected region of the $z$-plane containing the origin, and the multiplicity of $(z-t)^{\lambda-1}$ is removed by requiring $\log (z-\mathrm{t})$ to be real when $z-\mathrm{t}>0$ and $\Gamma$ is the Gamma function.

With the above definition, we know that

$$
\mathrm{D}_{z}^{-\lambda} \mathrm{f}(z)=\frac{1}{\Gamma(2+\lambda)} z^{1+\lambda}+\sum_{k=n+1}^{\infty} \frac{k !}{\Gamma(k+1+\lambda)} a_{k} z^{k+\lambda}
$$

for $\lambda>0$ and $f \in T_{n}$. Further applying the fractional integral for $f \in T_{n}$, we define a new operator $A_{-\lambda} f(z)$ given by

$$
A_{-\lambda} f(z)=\frac{\Gamma\left(\frac{3+\lambda}{2}\right)}{\Gamma\left(\frac{3-\lambda}{2}\right)} z^{\frac{1-\lambda}{2}} D_{z}^{-\lambda}\left(z^{\frac{-1-\lambda}{2}} f(z)\right)
$$


where $0 \leq \lambda \leq 1$. If $\lambda=0$, then (6) becomes $A_{0} f(z)=f(z)$ and if $\lambda=1$, then (6) leads us that

$$
A_{-1} f(z)=D_{z}^{-1}\left(\frac{f(z)}{z}\right)=\int_{0}^{z} \frac{f(t)}{t} d t
$$

With this integral operator, we know

$$
A_{-j-\lambda} f(z)=A_{-j}\left(A_{-\lambda} f(z)\right)
$$

where $j \in \mathbb{N}$ and $0 \leq \lambda \leq 1$. This operator $A_{-j-\lambda} f(z)$ is the generalization of the Alexander integral operator $A_{-1} f(z)$. Here, we note that

$$
A_{-\lambda} f(z)=z+\sum_{k=n+1}^{\infty} \frac{\Gamma\left(\frac{3+\lambda}{2}\right) \Gamma\left(\frac{2 k+1-\lambda}{2}\right)}{\Gamma\left(\frac{3-\lambda}{2}\right) \Gamma\left(\frac{2 k+1+\lambda}{2}\right)} a_{k} z^{k}
$$

and

$$
A_{-j-\lambda} f(z)=z+\sum_{k=n+1}^{\infty} \frac{\Gamma\left(\frac{3+\lambda}{2}\right) \Gamma\left(\frac{2 k+1-\lambda}{2}\right)}{\Gamma\left(\frac{3-\lambda}{2}\right) \Gamma\left(\frac{2 k+1+\lambda}{2}\right) k^{j}} a_{k} z^{k}
$$

where $j \in \mathbb{N}$ and $0 \leq \lambda \leq 1$. From the above, we know that

$$
A_{-j-\lambda} f(z)=A_{-j}\left(A_{-\lambda} f(z)\right)=A_{-\lambda}\left(A_{-j} f(z)\right)
$$

for $f \in T_{n}$. For $m$ different boundary points $z_{s}(s=1,2,3, \ldots, m)$ with $\left|z_{s}\right|=1$, we consider

$$
\alpha_{m}=\frac{1}{m} \sum_{s=1}^{m} \frac{A_{-j-\lambda} f\left(z_{s}\right)}{z_{s}}
$$

where $\alpha_{m} \in e^{i \beta} A_{-j-\lambda} f(\mathbb{U}), \alpha_{m} \neq 1$ and $-\frac{\pi}{2} \leq \beta \leq \frac{\pi}{2}$. For such $\alpha_{m}$, if $f \in T_{n}$ satisfies

$$
\left|\frac{e^{i \beta \frac{A_{-j-\lambda} f(z)}{z}}-\alpha_{m}}{e^{i \beta}-\alpha_{m}}-1\right|<\rho, \quad z \in \mathbb{U}
$$

for some real $\rho>0$, we say that the function $f$ belongs to the subclass $B_{n}\left(\alpha_{m}, \beta, \rho ; j, \lambda\right)$ of $T_{n}$. With this definition for the class $B_{n}\left(\alpha_{m}, \beta, \rho ; j, \lambda\right)$, we see that the condition (13) is equivalent to

$$
\left|\frac{A_{-j-\lambda} f(z)}{z}-1\right|<\rho\left|e^{i \beta}-\alpha_{m}\right|, \quad z \in \mathbb{U} .
$$


If we consider the function $f \in T_{n}$ given by

$$
f(z)=z+\frac{\Gamma\left(\frac{3-\lambda}{2}\right) \Gamma\left(\frac{2 n+3+\lambda}{2}\right)}{\Gamma\left(\frac{3+\lambda}{2}\right) \Gamma\left(\frac{2 n+3-\lambda}{2}\right)} \rho\left(e^{i \beta}-\alpha_{m}\right)(n+1)^{j} z^{n+1}
$$

then $f$ satisfies

$$
\left|\frac{A_{-j-\lambda} f(z)}{z}-1\right|=\rho\left|e^{i \beta}-\alpha_{m}\right||z|^{n}<\rho\left|e^{i \beta}-\alpha_{m}\right|, \quad z \in \mathbb{U} .
$$

Therefore, $f$ given by (15) belongs to the class $B_{n}\left(\alpha_{m}, \beta, \rho ; j, \lambda\right)$.

Discussing our problems for $f \in B_{n}\left(\alpha_{m}, \beta, \rho ; j, \lambda\right)$, we have to introduce the following lemma due to S. S. Miller and P. T. Mocanu [5, 6] (also, due to I. S. Jack [3]).

Lemma 1 Let the function $w$ given by

$$
w(z)=a_{n} z^{n}+a_{n+1} z^{n+1}+a_{n+2} z^{n+2}+\ldots, \quad(n \in \mathbb{N})
$$

be analytic in $\mathbb{U}$ with $w(0)=0$. If $|w(z)|$ attains its maximum value on the circle $|z|=\mathrm{r}$ at a point $z_{0},\left(0<\left|z_{0}\right|<1\right)$ then there exists a real number $\mathrm{k} \geq \mathrm{n}$ such that

$$
\frac{z_{0} w^{\prime}\left(z_{0}\right)}{w\left(z_{0}\right)}=k
$$

and

$$
\operatorname{Re}\left(1+\frac{z_{0} w^{\prime \prime}\left(z_{0}\right)}{w^{\prime}\left(z_{0}\right)}\right) \geq k
$$

\section{Properties of functions concerning with the class $B_{n}\left(\alpha_{m}, \beta, \rho ; j, \lambda\right)$}

Our first property for $f \in T_{n}$ is as follows.

Theorem 1 If $\mathrm{f} \in \mathrm{T}_{\mathrm{n}}$ satisfies

$$
\left|\frac{A_{-j-\lambda+1} f(z)}{A_{-j-\lambda} f(z)}-1\right|<\frac{\left|e^{i \beta}-\alpha_{m}\right| n \rho}{1+\left|e^{i \beta}-\alpha_{m}\right| \rho}, \quad z \in \mathbb{U}
$$

for some $\alpha_{\mathrm{m}}$ defined by (12) with $\alpha_{\mathrm{m}} \neq 1$ such that $z_{\mathrm{s}} \in \partial \mathbb{U}(\mathrm{s}=1,2,3, \ldots, \mathrm{m})$, and for some real $\rho>1$, then

$$
\left|\frac{A_{-j-\lambda} f(z)}{z}-1\right|<\rho\left|e^{i \beta}-\alpha_{m}\right|, \quad z \in \mathbb{U}
$$

that is, $f \in \mathrm{B}_{\mathfrak{n}}\left(\alpha_{\mathrm{m}}, \beta, \rho ; j, \lambda\right)$. 
Proof. We introduce the function $w$ by

$w(z)=\frac{e^{i \beta} \frac{A_{-j-\lambda} f(z)}{z}-\alpha_{m}}{e^{i \beta}-\alpha_{m}}-1=\frac{e^{i \beta}}{e^{i \beta}-\alpha_{m}}\left\{\sum_{k=n+1}^{\infty} \frac{\Gamma\left(\frac{3+\lambda}{2}\right) \Gamma\left(\frac{2 k+1-\lambda}{2}\right)}{\Gamma\left(\frac{3-\lambda}{2}\right) \Gamma\left(\frac{2 k+1+\lambda}{2}\right) k^{j}} a_{k} z^{k-1}\right\}$.

Then, $w$ is analytic in $\mathbb{U}$ with $w(0)=0$ and

$$
\frac{A_{-j-\lambda} f(z)}{z}=1+\left(1-e^{-i \beta} \alpha_{m}\right) w(z) .
$$

It follows from the above that

$$
\frac{z\left(A_{-j-\lambda} f(z)\right)^{\prime}}{A_{-j-\lambda} f(z)}-1=\frac{\left(1-e^{-i \beta} \alpha_{m}\right) z w^{\prime}(z)}{1+\left(1-e^{-i \beta} \alpha_{m}\right) w(z)} .
$$

Note that

$$
z\left(A_{-j-\lambda} f(z)\right)^{\prime}=A_{-j-\lambda+1} f(z) .
$$

So, (24) is the same as

$$
\frac{A_{-j-\lambda+1} f(z)}{A_{-j-\lambda} f(z)}-1=\frac{\left(1-e^{-i \beta} \alpha_{m}\right) z w^{\prime}(z)}{1+\left(1-e^{-i \beta} \alpha_{m}\right) w(z)} .
$$

Thus, our condition (20) gives that

$$
\left|\frac{A_{-j-\lambda+1} f(z)}{A_{-j-\lambda} f(z)}-1\right|=\left|\frac{\left(1-e^{-i \beta} \alpha_{m}\right) z w^{\prime}(z)}{1+\left(1-e^{-i \beta} \alpha_{m}\right) w(z)}\right|<\frac{\left|e^{i \beta}-\alpha_{m}\right| n \rho}{1+\left|e^{i \beta}-\alpha_{m}\right| \rho} .
$$

Now, we suppose that there exists a point $z_{0},\left(0<\left|z_{0}\right|<1\right)$ such that

$$
\max \left\{|w(z)| ;|z| \leq\left|z_{0}\right|\right\}=\left|w\left(z_{0}\right)\right|=\rho>1 .
$$

Then, we can write that $w\left(z_{0}\right)=\rho e^{i \theta},(0 \leq \theta \leq 2 \pi)$ and $z_{0} w^{\prime}\left(z_{0}\right)=k w\left(z_{0}\right)$, $(k \geq n)$ by Lemma 1 . For such a point $z_{0},\left(0<\left|z_{0}\right|<1\right)$ we see that

$$
\begin{aligned}
\left|\frac{A_{-j-\lambda+1} f\left(z_{0}\right)}{A_{-j-\lambda} f\left(z_{0}\right)}-1\right|= & \left|\frac{\left(1-e^{-i \beta} \alpha_{m}\right) z_{0} w^{\prime}\left(z_{0}\right)}{1+\left(1-e^{-i \beta} \alpha_{m}\right) w\left(z_{0}\right)}\right| \\
= & \left|\frac{\left(1-e^{-i \beta} \alpha_{m}\right) k \rho}{1+\left(1-e^{-i \beta} \alpha_{m}\right) \rho e^{i \theta}}\right| \\
& \geq \frac{\left|1-e^{-i \beta} \alpha_{m}\right| n \rho}{1+\left|1-e^{-i \beta} \alpha_{m}\right| \rho} \\
& =\frac{\left|e^{i \beta}-\alpha_{m}\right| n \rho}{1+\left|e^{i \beta}-\alpha_{m}\right| \rho} .
\end{aligned}
$$


Since (29) contradicts our condition (20), we know that there is no $z_{0}$, $\left(0<\left|z_{0}\right|<1\right)$ such that $\left|w\left(z_{0}\right)\right|=\rho>1$. Therefore, using $|w(z)|<\rho$ for all $z \in \mathbb{U}$, we have that

$$
|w(z)|=\left|\frac{e^{i \beta}\left(\frac{A_{-j-\lambda} f(z)}{z}-1\right)}{e^{i \beta}-\alpha_{m}}\right|<\rho, \quad z \in \mathbb{U},
$$

that is, that

$$
\left|\frac{A_{-j-\lambda} f(z)}{z}-1\right|<\rho\left|e^{i \beta}-\alpha_{m}\right|, \quad z \in \mathbb{U} .
$$

This completes the proof of the theorem.

Example 1 We consider the function $\mathrm{f} \in \mathrm{T}_{\mathrm{n}}$ given by

$$
f(z)=z+a_{n+1} z^{n+1}, \quad z \in \mathbb{U} .
$$

Then, we see that

$$
\begin{gathered}
\left|\frac{A_{-j-\lambda+1} f(z)}{A_{-j-\lambda} f(z)}-1\right|=\left|\frac{P(n, j, \lambda) n a_{n+1} z^{n}}{1+P(n, j, \lambda) a_{n+1} z^{n}}\right| . \\
\quad<\frac{n P(n, j, \lambda)\left|a_{n+1}\right|}{1-P(n, j, \lambda)\left|a_{n+1}\right|}, \quad z \in \mathbb{U}
\end{gathered}
$$

where

$$
0<\left|a_{n+1}\right|<\frac{1-P(n, j, \lambda)}{P(n, j, \lambda)}
$$

and

$$
P(n, j, \lambda)=\frac{\Gamma\left(\frac{3+\lambda}{2}\right) \Gamma\left(\frac{2 n+3-\lambda}{2}\right)}{\Gamma\left(\frac{3-\lambda}{2}\right) \Gamma\left(\frac{2 n+3+\lambda}{2}\right)(n+1)^{j}} .
$$

Now, we consider five boundary points

$$
\begin{aligned}
& z_{1}=e^{-i \frac{\arg \left(a_{n+1}\right)}{n}} \\
& z_{2}=e^{i \frac{\pi-6 \arg \left(a_{n+1}\right)}{6 n}} \\
& z_{3}=e^{i \frac{\pi-4 \arg \left(a_{n+1}\right)}{4 n}} \\
& z_{4}=e^{i \frac{\pi-3 \arg \left(a_{n+1}\right)}{3 n}}
\end{aligned}
$$


and

$$
z_{5}=e^{i \frac{\pi-2 \arg \left(a_{n+1}\right)}{2 n}} .
$$

For such $z_{s}(s=1,2,3,4,5)$, we have that

$$
\begin{gathered}
\frac{A_{-j-\lambda} f\left(z_{1}\right)}{z_{1}}=1+P(n, j, \lambda)\left|a_{n+1}\right|, \\
\frac{A_{-j-\lambda} f\left(z_{2}\right)}{z_{2}}=1+P(n, j, \lambda)\left|a_{n+1}\right| \frac{\sqrt{3}+i}{2}, \\
\frac{A_{-j-\lambda} f\left(z_{3}\right)}{z_{3}}=1+P(n, j, \lambda)\left|a_{n+1}\right| \frac{\sqrt{2}(1+i)}{2}, \\
\frac{A_{-j-\lambda} f\left(z_{4}\right)}{z_{4}}=1+P(n, j, \lambda)\left|a_{n+1}\right| \frac{1+\sqrt{3} i}{2},
\end{gathered}
$$

and

$$
\frac{A_{-j-\lambda} f\left(z_{5}\right)}{z_{5}}=1+P(n, j, \lambda)\left|a_{n+1}\right| i .
$$

It follows from the above that

$$
\alpha_{5}=\frac{1}{5} \sum_{s=1}^{5} \frac{A_{-j-\lambda} f\left(z_{s}\right)}{z_{s}}=1+\frac{(3+\sqrt{2}+\sqrt{3}) P(n, j, \lambda)\left|a_{n+1}\right|(1+i)}{10}
$$

and that

$$
\left|1-e^{-i \beta} \alpha_{5}\right|=\frac{\sqrt{2}(3+\sqrt{2}+\sqrt{3}) P(n, j, \lambda)\left|a_{n+1}\right|}{10}
$$

with $\beta=0$. For such $\alpha_{5}$ and $\beta$, we consider $\rho>1$ with

$$
\frac{n P(n, j, \lambda)\left|a_{n+1}\right|}{1-P(n, j, \lambda)\left|a_{n+1}\right|} \leq \frac{\left|e^{i \beta}-\alpha_{5}\right| n \rho}{1+\left|e^{i \beta}-\alpha_{5}\right| \rho} \text {. }
$$

This gives us that

$$
\rho \geq \frac{10}{\sqrt{2}(3+\sqrt{2}+\sqrt{3})\left(1-\left(1+\left|a_{n+1}\right|\right) P(n, j, \lambda)\right)}>\frac{10}{\sqrt{2}(3+\sqrt{2}+\sqrt{3})}>1 .
$$

For such $\alpha_{5}$ and $\rho>1$, the function $\mathrm{f}$ satisfies

$$
\left|\frac{A_{-j-\lambda} f(z)}{z}-1\right|<P(n, j, \lambda)\left|a_{n+1}\right| \leq \rho\left|e^{i \beta}-\alpha_{5}\right|, \quad z \in \mathbb{U} .
$$


Next, we derive the following theorem.

Theorem 2 If $\mathrm{f} \in \mathrm{T}_{\mathrm{n}}$ satisfies

$$
\left|\left(\frac{A_{-j-\lambda+1} f(z)}{A_{-j-\lambda} f(z)}-1\right)\left(\frac{A_{-j-\lambda} f(z)}{z}-1\right)\right|<\frac{\left|e^{i \beta}-\alpha_{m}\right|^{2} n \rho^{2}}{1+\left|e^{i \beta}-\alpha_{m}\right| \rho}, \quad z \in \mathbb{U}
$$

for some $\alpha_{m}$ defined by (12) with $\alpha_{m} \neq 1$ and for some real $\rho>1$, then

$$
\left|\frac{A_{-j-\lambda} f(z)}{z}-1\right|<\rho\left|e^{i \beta}-\alpha_{m}\right|, \quad z \in \mathbb{U}
$$

that is, $f \in \mathrm{B}_{\mathfrak{n}}\left(\alpha_{\mathrm{m}}, \beta, \rho ; j, \lambda\right)$.

Proof. Define the function $w$ by (22). Applying (25), our condition (50) leads us that

$$
\begin{aligned}
\left|\left(\frac{A_{-j-\lambda+1} f(z)}{A_{-j-\lambda} f(z)}-1\right)\left(\frac{A_{-j-\lambda} f(z)}{z}-1\right)\right| & =\left|\frac{\left(1-e^{-i \beta} \alpha_{m}\right)^{2} z w(z) w^{\prime}(z)}{1+\left(1-e^{-i \beta} \alpha_{m}\right) w(z)}\right| \\
& \leq \frac{\left|e^{i \beta}-\alpha_{m}\right|^{2} n \rho^{2}}{1+\left|e^{i \beta}-\alpha_{m}\right| \rho}, \quad z \in \mathbb{U} .
\end{aligned}
$$

Suppose that there exists a point $z_{0},\left(0<\left|z_{0}\right|<1\right)$ such that

$$
\max \left\{|w(z)| ;|z| \leq\left|z_{0}\right|\right\}=\left|w\left(z_{0}\right)\right|=\rho>1 .
$$

Then, applying Lemma 1 , we write that $w\left(z_{0}\right)=\rho e^{i \theta},(0 \leq \theta \leq 2 \pi)$ and $z_{0} w^{\prime}\left(z_{0}\right)=k w\left(z_{0}\right),(k \geq n)$. This shows us that

$$
\begin{aligned}
\left|\left(\frac{A_{-j-\lambda+1} f(z)}{A_{-j-\lambda} f(z)}-1\right)\left(\frac{A_{-j-\lambda} f(z)}{z}-1\right)\right| & =\left|\frac{\left(1-e^{-i \beta} \alpha_{m}\right)^{2} z_{0} w\left(z_{0}\right) w^{\prime}\left(z_{0}\right)}{1+\left(1-e^{-i \beta} \alpha_{m}\right) w\left(z_{0}\right)}\right| \\
& =\frac{\left|e^{i \beta}-\alpha_{m}\right|^{2} \rho^{2} k}{\left|1+\left(1-e^{-i \beta} \alpha_{m}\right) \rho e^{i \theta}\right|} \\
& \geq \frac{\left|e^{i \beta}-\alpha_{m}\right|^{2} n \rho^{2}}{1+\left|e^{i \beta}-\alpha_{m}\right| \rho}
\end{aligned}
$$

which contradicts our condition (50). Thus there is no $z_{0},\left(0<\left|z_{0}\right|<1\right)$ such that $\left|w\left(z_{0}\right)\right|=\rho>1$. This shows us that

$$
\left|\left(\frac{A_{-j-\lambda} f(z)}{z}-1\right)\right|<\rho\left|e^{i \beta}-\alpha_{m}\right|, \quad z \in \mathbb{U} .
$$


Example 2 Consider a function $\mathrm{f} \in \mathrm{T}_{\mathrm{n}}$ given by

$$
\mathrm{f}(z)=z+\mathrm{a}_{\mathrm{n}+1} z^{\mathrm{n}+1}, z \in \mathbb{U}
$$

with $0<\left|\mathrm{a}_{\mathrm{n}+1}\right|<\frac{1}{\mathrm{P}(\mathrm{n}, \mathrm{j}, \lambda)}$, where $\mathrm{P}(\mathrm{n}, \mathrm{j}, \lambda)$ is given by (35). It follows that

$$
\begin{aligned}
\left|\left(\frac{A_{-j-\lambda+1} f(z)}{A_{-j-\lambda} f(z)}-1\right)\left(\frac{A_{-j-\lambda} f(z)}{z}-1\right)\right|= & \left|\frac{n P(n, j, \lambda)^{2} a_{n+1}^{2} z^{2 n}}{1+P(n, j, \lambda) a_{n+1} z^{n}}\right| \\
& <\frac{n P(n, j, \lambda)^{2}\left|a_{n+1}\right|^{2}}{1-P(n, j, \lambda)\left|a_{n+1}\right|}, \quad z \in \mathbb{U} .
\end{aligned}
$$

Considering five boundary points $z_{1}, z_{2}, z_{3}, z_{4}$ and $z_{5}$ in Example 1, we see that

$$
\left|e^{i \beta}-\alpha_{5}\right|=\frac{\sqrt{2}(3+\sqrt{2}+\sqrt{3}) P(n, j, \lambda)\left|a_{n+1}\right|}{10}
$$

with $\beta=0$. If we consider $\rho>1$ such that

$$
\frac{n P(n, j, \lambda)^{2}\left|a_{n+1}\right|^{2}|z|}{1-P(n, j, \lambda)\left|a_{n+1}\right|} \leq \frac{\left|e^{i \beta}-\alpha_{5}\right|^{2} n \rho^{2}}{1+\left|e^{i \beta}-\alpha_{5}\right| \rho},
$$

then $\rho$ satisfies

$$
\rho \geq \frac{10}{\sqrt{2}(3+\sqrt{2}+\sqrt{3}) P(n, j, \lambda)\left|a_{n+1}\right|}>1 .
$$

For such $\alpha_{5}$ and $\rho$, f satisfies

$$
\left|\frac{A_{-j-\lambda} f(z)}{z}-1\right|<P(n, j, \lambda)\left|a_{n+1}\right| \leq \rho\left|e^{i \beta}-\alpha_{5}\right|, \quad z \in \mathbb{U} .
$$

Our next result reads as follows.

Theorem 3 If $\mathrm{f} \in \mathrm{T}_{\mathrm{n}}$ satisfies

$$
\left|\frac{A_{-j-\lambda+p} f(z)}{z}-1\right|<\rho\left|e^{i \beta}-\alpha_{m}\right|(n+1), \quad z \in \mathbb{U} .
$$

for some $\alpha_{m}$ defined by (12) with $\alpha_{m} \neq 1$ and for some real $\rho>1$, then

$$
\left|\frac{A_{-j-\lambda+p-1} f(z)}{z}-1\right|<\rho\left|e^{i \beta}-\alpha_{m}\right|, \quad z \in \mathbb{U}
$$

where $p=0,1,2, \ldots, j$. 
Proof. We consider the function $w$ defined by

$$
\begin{aligned}
w(z) & =\frac{e^{i \beta} \frac{A_{-j-\lambda+p-1} f(z)}{z}-\alpha_{m}}{e^{i \beta}-\alpha_{m}}-1 \\
& =\frac{e^{i \beta}}{e^{i \beta}-\alpha_{m}}\left\{\sum_{k=n+1}^{\infty} \frac{\Gamma\left(\frac{3+\lambda}{2}\right) \Gamma\left(\frac{2 k+1-\lambda}{2}\right)}{\Gamma\left(\frac{3-\lambda}{2}\right) \Gamma\left(\frac{2 k+1+\lambda}{2}\right) k^{j-p+1}} a_{k} z^{k-1}\right\} .
\end{aligned}
$$

Thus $w$ is analytic in $\mathbb{U}, w(0)=0$, and

$$
A_{-j-\lambda+p-1} f(z)=z+\left(1-e^{-i \beta} \alpha_{m}\right) z w(z) .
$$

Noting that

$$
\begin{aligned}
A_{-j-\lambda+p} f(z) & =z\left(A_{-j-\lambda+p-1} f(z)\right)^{\prime} \\
& =z\left\{1+\left(1-e^{-i \beta} \alpha_{m}\right) w(z)\left(1+\frac{z w^{\prime}(z)}{w(z)}\right)\right\},
\end{aligned}
$$

we have that

$$
\begin{aligned}
\left|\frac{A_{-j-\lambda+p} f(z)}{z}-1\right|= & \left|1-e^{-i \beta} \alpha_{m}\right||w(z)|\left|1+\frac{z w^{\prime}(z)}{w(z)}\right| \\
& <\rho\left|e^{i \beta}-\alpha_{m}\right|(n+1), \quad z \in \mathbb{U}
\end{aligned}
$$

by the condition (62). Suppose that there exists a point $z_{0},\left(0<\left|z_{0}\right|<1\right)$ such that

$$
\max \left\{|w(z)| ;|z| \leq\left|z_{0}\right|\right\}=\left|w\left(z_{0}\right)\right|=\rho>1 .
$$

Then, letting $w\left(z_{0}\right)=\rho e^{i \theta},(0 \leq \theta \leq 2 \pi)$ and $z_{0} w^{\prime}\left(z_{0}\right)=k w\left(z_{0}\right),(k \geq n)$ with Lemma 1 , we see that

$$
\left|\frac{A_{-j-\lambda+p} f\left(z_{0}\right)}{z_{0}}-1\right|=\rho\left|e^{i \beta}-\alpha_{m}\right|(k+1) \geq \rho\left|e^{i \beta}-\alpha_{m}\right|(n+1) .
$$

This contradicts the inequality (67). Therefore, we don't have any $z_{0} \in \mathbb{U}$ such that $\left|w\left(z_{0}\right)\right|=\rho>1$. This shows us that

$$
|w(z)|=\left|\frac{\alpha_{m}}{e^{i \beta}-\alpha_{m}}\left(\frac{A_{-j-\lambda+p-1} f(z)}{z}-1\right)\right|<\rho, \quad z \in \mathbb{U},
$$

that is, that

$$
\left|\frac{A_{-j-\lambda+p-1} f(z)}{z}-1\right|<\rho\left|e^{i \beta}-\alpha_{m}\right|, \quad z \in \mathbb{U} .
$$

This completes the proof of our theorem. 
Corollary 1 If $\mathrm{f} \in \mathrm{T}_{\mathrm{n}}$ satisfies

$$
\left|\frac{A_{-j-\lambda+p} f(z)}{z}-1\right|<\rho\left|e^{i \beta}-\alpha_{m}\right|(n+1)^{p}, \quad z \in \mathbb{U}
$$

for some $\alpha_{m}$ given by (12) with $\alpha_{m} \neq 1$, and for some real $\rho>1$, then

$$
\left|\frac{A_{-j-\lambda} f(z)}{z}-1\right|<\rho\left|e^{i \beta}-\alpha_{m}\right|, \quad z \in \mathbb{U}
$$

where $p=0,1,2, \ldots, j$.

Proof. With Theorem 3, we say that if $\mathrm{f} \in \mathrm{T}_{\mathrm{n}}$ satisfies

$$
\left|\frac{A_{-j-\lambda+p} f(z)}{z}-1\right|<\rho\left|e^{i \beta}-\alpha_{m}\right|(n+1)^{p}, \quad z \in \mathbb{U},
$$

then

$$
\left|\frac{A_{-j-\lambda+p-1} f(z)}{z}-1\right|<\rho\left|e^{i \beta}-\alpha_{m}\right|(n+1)^{p-1}, \quad z \in \mathbb{U} .
$$

Further, we have that

$$
\left|\frac{A_{-j-\lambda+p-2} f(z)}{z}-1\right|<\rho\left|e^{i \beta}-\alpha_{m}\right|(n+1)^{p-2}, \quad z \in \mathbb{U},
$$

from (75). Finally, we obtain that

$$
\left|\frac{A_{-j-\lambda} f(z)}{z}-1\right|<\rho\left|e^{i \beta}-\alpha_{m}\right|, \quad z \in \mathbb{U} .
$$

Example 3 Consider the function $\mathrm{f} \in \mathrm{T}_{\mathrm{n}}$ given by

$$
f(z)=z+a_{\mathfrak{n}+1} z^{\mathfrak{n}+1}, z \in \mathbb{U} .
$$

Since

$$
A_{-j-\lambda+p} f(z)=z+\frac{\Gamma\left(\frac{3+\lambda}{2}\right) \Gamma\left(\frac{2 n+3-\lambda}{2}\right)}{\Gamma\left(\frac{3-\lambda}{2}\right) \Gamma\left(\frac{2 n+3+\lambda}{2}\right)(n+1)^{j-p+2}} a_{n+1} z^{n+1},
$$

we have

$$
\left|\frac{A_{-j-\lambda+p} f(z)}{z}-1\right|=\left|P(n, j, \lambda)(n+1)^{p-2} a_{n+1} z^{n}\right|<P(n, j, \lambda)(n+1)^{p-2}\left|a_{n+1}\right|
$$


where

$$
0<\left|a_{n+1}\right|<\frac{1}{P(n, j, \lambda)}
$$

and $\mathrm{P}(\mathrm{n}, \mathrm{j}, \lambda)$ is given by $(35)$.

Consider five boundary points $z_{1}, z_{2}, z_{3}, z_{4}$ and $z_{5}$ in Example 1 . Then $\alpha_{5}$ satisfies (46) and $\left|1-e^{-i \beta} \alpha_{5}\right|$ satisfies (47) for $\beta=0$. For such $\alpha_{5}$ and $\beta$, we consider $\rho>1$ by

$$
\left|\frac{A_{-j-\lambda+p} f(z)}{z}-1\right|<P(n, j, \lambda)(n+1)^{p-2}\left|a_{n+1}\right| \leq \rho\left|e^{i \beta}-\alpha_{5}\right|(n+1)^{p-2}, \quad z \in \mathbb{U},
$$

Then $\rho$ satisfies

$$
\rho \geq \frac{P(n, j, \lambda)\left|a_{n+1}\right|}{\left|e^{i \beta}-\alpha_{5}\right|}=\frac{10}{\sqrt{2}(3+\sqrt{2}+\sqrt{3})}>1 .
$$

With the above $\alpha_{5}$ and $\rho$, we have

$$
\left|\frac{A_{-j-\lambda} f(z)}{z}-1\right|<P(n, j, \lambda)\left|a_{n+1}\right| \leq \rho\left|e^{i \beta}-\alpha_{5}\right|, \quad z \in \mathbb{U} .
$$

\section{References}

[1] M. Acu, Some preserving properties of the generalized Alexander integral operator, General Math., 10 (2002), 37-46.

[2] J. W. Alexander, Functions which map the interior of the unit circle upon simple regions, Ann. of Math., Second Series, 17 (1915),12-22.

[3] I. S. Jack, Functions starlike and convex of order $\alpha$, J. London Math. Soc., 2 (1971), 469-474.

[4] K. Kugita, K. Kuroki and S. Owa, On $(\alpha, \delta)$-neighborhood defined by a new operator for certain analytic functions, Far East J. Math. Sci., 47 (2010), 1-12.

[5] S. S. Miller and P. T. Mocanu, Second order differential inequalities in the complex plane, J. Math. Anal. Appl., 65 (1978), 289-305.

[6] S. S. Miller and P. T. Mocanu, Differential Subordinations: Theory and Applications, Marcel Dekker Inc. New York, (2000). 
[7] S. Owa, On the distortion theorems I, Kyungpook Math. J., 18 (1978), $53-59$.

[8] S. Owa and H. M. Srivastava, Univalent and starlike generalized hypergeometric functions, Canad. J. Math., 39 (1987), 1057-1077.

Received: April 29, 2020 